\title{
Sonderkurse der Ärztlichen Schule der SMS
}

Online publiziert: 26. Mai 2021

๑ Springer Medizin Verlag GmbH, ein Teil von Springer Nature 2021

(ALLE Kurse werden anerkannt für den CPC - Certified Physician of Chinese Medicine und sind akkreditiert für die CME-Punkte)

\section{Juli/August}

\section{„SMS Summerschool“ (nur für Medizinstudenten!)}

voraussichtlich 31.07.-04.08.2021 in Tschagguns, Österreich

4-Tages-Kurs I Dozenten: Dr. Marc Scheuermann et al.

Was kann ich mit Akupunktur \& Chinesischer Medizin alles behandeln?

Wir geben Dir spannende Einblicke in die Diagnostik und Therapie der Chinesischen Medizin. Wir werden viel praktisch üben, Fälle und klinische Situationen simulieren, Akupunkturpunkte lokalisieren, Nadeln setzen, Schröpfen, Moxen, Kräuter kochen und trinken, Tuina Bausteine an uns üben, Taiji auf der Wiese machen, meditieren, reden, lachen, diskutieren, den wunderschönen Ort und das tolle Bio-Essen genießen, ev. auch ein bisschen wandern ...

\section{September}

Start neuer Ausbildungszyklus Diagnostik \& Physiologie Entscheidend für die praktische Ausübung der Chinesischen Medizin ist das andersartige Verständnis vom menschlichen Körper sowie dessen Reaktionen auf unterschiedlichste Einflüsse und Krankheiten, welche sich daraus entwickeln können.

Als regulärer Ausbildungszyklus mit 9 Wochenendkursen in Hamburg

Start mit Kurs 1 „Diagnostik 1 \& 2“ am 18./19.09.2021

2-Tages-Kurs । 16 UE । ca. 16 CME-Punkte | Dozent: Dr. Werner Jansen
Ausbildungszyklus Arzneitherapie, Start Block B Komplett ONLINE

Im Fortbildungs-Modul „Arzneitherapie“ werden die chinesischen Phytotherapeutika detailliert dargestellt. Die ausführliche Erörterung wichtiger Kombinationen und klassischer Rezepturen runden das jeweilige Thema ab. Dieser Zyklus vermittelt fundierte Kenntnisse des wichtigsten Therapieverfahrens der Chinesischen Medizin und ermöglicht eine differenzierte Anwendung in der Praxis.

Start mit Kurs 1B am 25./26.09.2021

2-Tages-Kurs I 16 UE I ca. 16 CME-Punkte I Dozent: Dr. Josef Hummelsberger

\section{Oktober}

„East meets West“" - Diagnostik, Mobilisationen und Manipulationen am Bewegungsapparat

02./03.10.2021 in Hamburg

1,5-Tages-Kurs I Dozentin: Sabine Zeitler

Für den gezielteren Einsatz mobilisierender und manipulierender Tuina-Techniken an Wirbelsäule und Extremitätengelenken ist eine sichere Handhabung von diagnostischen Tests am Bewegungsapparat sinnvoll. Diese Testverfahren aus der Manuellen Medizin und der Osteopathie helfen bei der Auswahl der erlernten Tuina-Techniken.

In diesem Seminar werden diagnostische Testverfahren an der Wirbelsäule und den wichtigsten Extremitätengelenken gezeigt und geübt. Zudem widmen wir uns dem Vergleich von Mobilisations- und Manipulationstechniken der Tuina zu westlichen Mobilisations- und Manipulationstechniken aus der Manuellen Therapie und Osteopathie. 
Schwerpunkte:

- Wirbelsäule (HWS, BWS, LWS)

- Schulter

- Hüftgelenk und Becken (ISG)

- Gelenkmobilisationen und Manipulationen (Tuina vs. Osteopathie)

\section{Klinische Woche ,Kreta ${ }^{66}$}

17.-22.10.2021 in Falassarna, Griechenland

6-Tages-Kurs I Dozenten: Dres. Josef Hummelsberger, Alexander Simon

Diese Woche wird sehr praktisch-klinisch ausgerichtet, mit vielen Fallbeispielen und Einüben des differentialdiagnostischen Vorgehens. Wir wollen besonders ausführlich vormittags wie nachmittags Fälle diskutieren und erarbeiten und würden auch die Teilnehmer bitten, kritische Fälle ,,mitzubringen“! Und Qigong am Morgen!

Schwerpunkte:

- Refluxkrankheit und GERD-Syndrome, PPI-Problematik

- schwierige neurologische Krankheiten: PNP, Restless legs, Post-Stroke, Tinnitus

- Atemwegskrankheiten/Asthma

- Akupunktur und Leitbahn-Praxis nach Wang Juyi und anderen

\section{November}

\section{Klinisches Seminar ,HNO-Erkrankungen“ \\ 05.-07.11.2021 in Zürich, Schweiz \\ 6-Tages-Kurs I Dozentin: Dr. Rose Fintelmann \\ Dieses Seminar vermittelt die klinische Vorgehensweise und Syndromdifferenzierung der Chinesischen Medizin in der HNO mit folgenden Schwerpunkten:}

- Sinusitis, Rhinitis und Nasenatmungsbehinderung

- Kinder in der HNO: Infekte und Adenoide

- Chronische Ohrprobleme wie Otitis externa, Belüftungsstörungen

- Hörsturz, Tinnitus und HNO-bedingter Schwindel

Es werden Behandlungsstrategien für Akupunktur und Chinesische Arzneitherapie bei diesen Krankheitsbildern entworfen, Fallbeispiele illustrieren exemplarische Behandlungsverläufe.

\section{Fortgeschrittenen Seminar,Wenbing“6 \\ 11.-14.11.2021 in Hamburg}

4-Tages-Kurs I Dozenten: Dres. Anke Iptchiler, Josef Hummelsberger

Durch Wärme (calor) ausgelöste Erkrankungen. Wenbing (morbi temperati) bezeichnet ein Konzept der Chinesischen
Medizin, das sich mit durch spezifische Schrägläufigkeiten (Heteropatien) ausgelösten Erkrankungen auseinandersetzt. Diese sind meist akut, infektiös und gehen mit Wärme-Zeichen (calor) einher. Nachdem die Wenbing-Lehre im 17. Jahrhundert entstanden war, erfuhr sie durch Ye Tianshi und Wu Jutong im 18. und 19. Jh. eine deutliche Ausarbeitung und Differenzierung. Zur Diagnose und Behandlung infektiöser Krankheiten stellt sie bis heute im ärztlichen Alltag eine wesentliche Erweiterung dar, gerade auch durch die Zunahme komplizierter Infektionserkrankungen und $\mathrm{Au}$ toimmunprozesse.

Wenbing ist auch die Basis für aktuelle Behandlungsoptionen mit Chinesischer Arzneitherapie für die COVID-19-Pandemie.

\section{Fortgeschrittenen Seminar ,Zungendiagnostik“6 \\ 27./28.11.2021 in Hamburg}

1,5-Tages-Kurs I Dozent: Dr. Christian Thede

Die Diagnose aus der Zunge dient vielfach der Sicherung einer aufgrund der Anamnese erhobenen Verdachtsdiagnose und kann oft sogar allein wegweisend bei der Erstellung einer Chinesischen Diagnose sein. In diesem vertiefenden Diagnostik-Seminar lernen Sie, Zungenzeichen und deren Veränderungen in Bezug auf Leitkriterien und Agenzien genau zu deuten. Mit Hilfe reichhaltigen Bildmaterials wird Ihnen die Befunderhebung im Rahmen der Zungendiagnostik demonstriert und systematisch der Weg zu einer chinesischen diagnostischen Aussage vermittelt.

Die erlernten Fertigkeiten werden anhand klinischer Beispiele und praktischer Übungen unter laufender Unterstützung und Anleitung gefestigt, so dass die hinzugewonnenen diagnostischen Fähigkeiten bereits unmittelbar nach dem Seminar Ihre Arbeit in Praxis und Klinik bereichern können. 